\title{
A modified version of regularized meshless method for three dimensional potential problem
}

\author{
Cheng-Yang Lai ${ }^{1}$, Kue-Hong Chen ${ }^{1 *}$, Sheng-Wei $\operatorname{Lin}^{1}$, Ren Liu $^{1}$ \\ ${ }^{1}$ Department of Civil Engineering, National Ilan University, Taiwan \\ *khc6177@niu.edu.tw (Corresponding author: K. H. Chen)
}

\begin{abstract}
In this study, three-dimensional potential problem is solved using a novel meshless method. Due to the singularity of the kernel functions, the diagonal terms of the influence matrices in the method of fundamental solutions (MFS) are unobtainable. A proposed approach in the literature of the past decade, namely regularized meshless method (RMM), is proposed to overcome such difficulties by using the proposed desingularization (subtracting and adding-back) technique. The main difficulty for the coincidence of the source and collocation points then disappears. However, the disadvantage of RMM is the order of precision of obtained solution is lower than other numerical methods. In this study, we present a novel technique to promote the order of precision of diagonal term; therefore, we can obtain a more precise solution. Finally, we introduce a typical 3-D numerical example to illustrate the technique. The numerical result is compared with those obtained by the RMM; and a more precise result is obtained.
\end{abstract}

Keywords: Method of fundamental solutions; Regularized meshless method; Desingularization; Diagonal terms.

\section{Introduction}

The method of fundamental solutions (MFS) [1] is a prospective meshless/meshfree boundary collocation method for the solution of real engineering problems. An increasing number of journal paper the growing attractiveness of the MFS. It was applied as a foolproof and accurate method to the solution of many engineering problems. In order to avoid the source singularity of fundamental solutions, the source points are distributed on the non-physical boundary (fictitious boundary) [2] outside the physical domain. Note that the kernel function is constituted of two-point function which is one kind of the radial basis functions (RBFs) [3] ; the independent variable of two-point function depends on point location only. A regular singularity-free formulation was obtained as a result, and realizes an attractive truly boundary type and mathematically simple meshfree method. The ease of implementation of the MFS makes it a designated numerical method in this study. However, because of the controversial artificial boundary (off-set boundary), which shift a distance from the real boundary, the MFS has not become a dominant numerical method. Notwithstanding its gain in singularity free, the influence matrix becomes ill-posed matrix [4] ; its result is very unstable since the condition number for the influence matrix becomes very large.

In 2005, Young, Chen, and Lee [5] developed a novel meshless method, namely the RMM, to overcome the drawback of MFS; it implement a subtracting and adding-back technique [6] to regularize the singularity of the kernel functions, and therefore the diagonal terms of influence matrices can be derived when the source points are located on the real boundary. Furthermore, unlike other meshless methods such as the boundary knot method (BKM) [7] which also places the source points on the physical boundary but results in a badly ill-conditioned interpolation matrix, the condition number of the coefficient matrix of the RMM remains gentle even with a large number of source nodes. A similar technique, namely singular boundary method (SBM), was proposed by Chen and his collaborators [8]. This SBM formulation keeps merits of RMM in which the source points can also be distributed on the physical boundary and makes it an advantage by using the single-layer fundamental solution. However the major drawback of the RMM or SBM is that the precision of solution is not enough for irregular domain problems due to the insufficient precision of the finite value of diagonal terms. In this study, we provide a novel technique to modify the diagonal coefficients of the coefficient matrix of the RMM; by introducing a defined auxiliary problem [9] in the technique, we can derive a more precise finite value of diagonal terms and can alleviate the ill condition of influence matrix.

The defined auxiliary problem is proposed a period of time [9]. The literature shows the meaning of the defined auxiliary problem and how to be applied it in a wide variety of problems. Due to the defined auxiliary problem and the original problem possess the same influence matrix under the distribution of the same mesh or source points. It's more important which the defined auxiliary problem has the analytical solution obtained by the complementary solutions, which satisfies the governing equation (G.E.) and it is similar to the real analytical solution of the original problem. Through the distinct identities, we can modify the diagonal coefficients of influence matrix by the known analytical solutions in the interesting domain of defined auxiliary problem; the obtained influence matrix can alleviate ill-posed, thus we can obtain a more excellent performance and higher convergence order than the RMM.

\section{Corresponding author: author@e-mail.org}




\section{Problem statement}

Consider the boundary value problem (BVP) on a general domain $\Omega \in R^{2}$ with mixed-type boundary conditions (B.C.s) as:

$$
\begin{gathered}
\mathcal{L}[u(x)]=0, x \text { in } \Omega \\
u(x)=\bar{u}(x), x \text { on } B_{1} \\
t(x)=\frac{\partial u(x)}{\partial n_{x}}=\bar{t}(x), x \text { on } B_{2}
\end{gathered}
$$

Where $\mathcal{L}$ is the linearly differential of second order operator, $u(x)$ is the solution, $\Omega$ is the computational domain of the problem. The boundary conditions are described as following: $B_{1}$ is the essential boundary (Dirichlet boundary) in which the potential is prescribed by $\bar{u}(x), B_{2}$ is the natural boundary (Neumann boundary) in which the normal derivative is prescribed as $\bar{t}(x) ; B_{1}$ and $B_{2}$ construct the whole boundary of the domain $\Omega$.

\section{Formulation}

\subsection{Representation for numerical solution of problem}

By employing radial basis function (RBF) concept, the representation of the solution for the interior problem can be approximated in terms of a set of interpolation functions as:

$$
\begin{aligned}
& u\left(x_{i}\right)=\sum_{j=1}^{N} A\left(x_{i}, s_{j}\right) \alpha_{j} \\
& t\left(x_{i}\right)=\sum_{j=1}^{N} B\left(x_{i}, s_{j}\right) \alpha_{j}
\end{aligned}
$$

where $x_{i}$ and $s_{j}$,respectively, represent $i$ th collocation point and $j$ th source point, $N$ is the number of source points and $\alpha_{j}$ is the generalized unknowns.

$$
\begin{gathered}
A\left(x_{i}, s_{j}\right)=\frac{y_{i} \bar{n}_{i}}{r^{3}} \\
B\left(x_{i}, s_{j}\right)=\frac{3 y_{i} y_{j} n_{i} \bar{n}_{j}}{r^{5}}-\frac{n_{i} \bar{n}_{i}}{r^{3}}
\end{gathered}
$$

where $r=\left|x_{i}-s_{j}\right|, B\left(x_{i}, s_{j}\right)=\frac{\partial A\left(x_{i}, s_{j}\right)}{\partial n_{x}}$, in which $n_{x}$ is the normal derivative of $u$ at collocation point $x_{i}, n_{k}$ is the $k$ th component of the outward normal vector at $s_{j} ; \bar{n}_{k}$ is the $k$ th component of the outward normal vector at $x_{i}$ and $y_{k}=x_{i}-s_{j}$.

\subsection{Adding-back technique of regularized meshless method}

When the collocation point $x_{i}$ approaches to the source point $s_{j}$, Equations (4) and (5) will become singular. Equations (4) and (5) for the interior problems need to be regularized by using special treatment of the desingularization of subtracting and adding-back technique [6] as follows

$$
\begin{aligned}
u\left(x_{i}\right)= & \sum_{j=1}^{N} A^{(i)}\left(s_{j}, x_{i}\right) \alpha_{j}-\sum_{j=1}^{N} A^{(e)}\left(s_{j}, x_{i}\right) \alpha^{i} \\
= & \sum_{j=1}^{i-1} A^{(i)}\left(s_{j}, x_{i}\right) \alpha_{j}+\sum_{j=i+1}^{N} A^{(i)}\left(s_{j}, x_{i}\right) \alpha_{j} \\
& +\left[\sum_{m=1}^{N} A^{(i)}\left(s_{m}, x_{i}\right)-A^{(i)}\left(s_{i}, x_{i}\right)\right] \alpha_{i}, x_{i} \in B \\
t\left(x_{i}\right)= & \sum_{j=1}^{N} B^{(i)}\left(s_{j}, x_{i}\right) \alpha_{j}-\sum_{j=1}^{N} B^{(e)}\left(s_{j}, x_{i}\right) \alpha_{i} \\
= & \sum_{j=1}^{i-1} B^{(i)}\left(s_{j}, x_{i}\right) \alpha_{j}+\sum_{j=i+1}^{N} B^{(i)}\left(s_{j}, x_{i}\right) \alpha_{j} \\
& -\left[\sum_{m=1}^{N} B^{(i)}\left(s_{m}, x_{i}\right)-B^{(i)}\left(s_{i}, x_{i}\right)\right] \alpha_{i}, x_{i} \in B
\end{aligned}
$$

in which

$$
\begin{aligned}
& \sum_{j=1}^{N} A^{(e)}\left(s^{j}, x^{i}\right)=0, x^{i} \in B \\
& \sum_{j=1}^{N} B^{(e)}\left(s^{j}, x^{i}\right)=0, x^{i} \in B
\end{aligned}
$$

which the superscript $(i)$ and $(e)$ denotes the interior and exterior domain, respectively. The detail derivations of Equations (10) and (11) are given in the literature [6]. The original singular terms of $A^{(i)}\left(s^{i}, x^{i}\right)$ and $B^{(i)}\left(s^{i}, x^{i}\right)$ in Equations (4) and (5), have been transformed into regular terms $\left[\sum_{m=1}^{N} A^{(i)}\left(s^{m}, x^{i}\right)-A^{(i)}\left(s^{i}, x^{i}\right)\right] \quad$ and $-\left[\sum_{m=1}^{N} B^{(i)}\left(s^{m}, x^{i}\right)-B^{(i)}\left(s^{i}, x^{i}\right)\right]$ in Equations (8) and (9), respectively. In which the terms of $\sum_{m=1}^{N} A^{(i)}\left(s^{m}, x^{i}\right)$ and $\sum_{m=1}^{N} B^{(i)}\left(s^{m}, x^{i}\right)$ are the adding-back terms and the terms of $A^{(i)}\left(s^{i}, x^{i}\right)$ and $B^{(i)}\left(s^{i}, x^{i}\right)$ are the subtracting terms in the two brackets for the special treatment technique. After using the desingularization of subtracting and adding-back technique [6] , we are able to remove the singularity and hypersingularity of the kernel functions. Therefore, the diagonal coefficients for the interior problems can be extracted out as: 


$$
\left\{u_{i}\right\}=\left[\begin{array}{cccc}
\sum_{m=1}^{N} a_{1, m}-a_{1,1} & a_{1,2} & \cdots & a_{1, N} \\
a_{2,1} & \sum_{m=1}^{N} a_{2, m}-a_{2,2} & \cdots & a_{2, N} \\
\vdots & \vdots & \ddots & \vdots \\
a_{N, 1} & a_{N, 2} & \cdots & \sum_{m=1}^{N} a_{N, m}-a_{N, N}
\end{array}\right]\left\{\alpha_{i}\right\}
$$

$$
\left\{t_{i}\right\}=\left[\begin{array}{cccc}
-\left(\sum_{m=1}^{N} b_{1, m}-b_{1,1}\right) & b_{1,2} & \cdots & b_{1, N} \\
b_{2,1} & -\left(\sum_{m=1}^{N} b_{2, m}-b_{2,2}\right) & \cdots & b_{2, N} \\
\vdots & \vdots & \ddots & \vdots \\
b_{N, 1} & b_{N, 2} & \cdots & -\left(\sum_{m=1}^{N} b_{N, m}-b_{N, N}\right)
\end{array}\right]\left\{\alpha_{,}\right\}
$$

\subsection{Modified technique for modifying diagonal term of influence matrics}

The precision of the resingularized techniques in the abovesection are not enough for irregular domain problems due to the insufficient accurancy in deriving valid value of diagonal terms of the influence matrices [5]. A modified technique is employed to modify the diagonal term of influence matrices in the Equations (12) and (13). By using the technique, we can obtain a more precise result in deriving a diagonal value of the influence matrix. The scheme of the modified technique is depicted as following section:

\subsubsection{Defining auxiliary problem}

(1) Specifying G.E., contour and B.C. type

The G.E., domain shape and B.C. type in the defined auxiliary problem is defined as:

$$
\begin{aligned}
& \mathcal{L}^{\prime}\left[u^{q}(x)\right]=0 \quad x \text { in } \Omega^{\prime} \\
& u^{q}(x)=\bar{u}^{q}(x), \text { on } B_{1}^{\prime} \\
& \frac{\partial u^{q}(x)}{\partial n_{x}}=t^{-q}(x), \text { on } B_{2}^{\prime}
\end{aligned}
$$

where $\mathcal{L}^{\prime}$ is the differential operator of $u^{q}(x), u^{q}(x)$ is the potential of auxiliary problems, $\Omega^{\prime}$ is the computational domain of the auxiliary problem. $B_{1}^{\prime}$ and $B_{2}^{\prime}$ construct the whole boundary of the domain $\Omega^{\prime} \cdot \bar{u}^{q}(x)$ and $\bar{t}^{q}(x)$ are a specifying function $u^{q}(x)$.

In order to appropriate the original problem in the Equations (1)-(3), we choose $\mathcal{L}^{\prime}=\mathcal{L}, \Omega^{\prime}=\Omega, B_{1}^{\prime}=B_{1}, B_{2}^{\prime}=B_{2}$, namely, the G.E., domain shape and B.C. type in the defined auxiliary problem is a duplicate of the original problem.

\section{(2) Giving quasi-analytical solution}

The potential, $u^{q}(x)$, in the auxiliary problem at arbitrary point $x$ in the domain is the linear combination of the complete set functions as follows:

$$
u^{q}(x)=\sum_{j=1}^{M} \Phi_{j}(x) c_{j}, x \text { in } \Omega^{\prime}
$$

where $\Phi_{j}(x)$ choose complementary solution sets which satisfies the G.E. in Equation (14), $M$ is the total number of the complementary solution set and $c_{j}$, denotes the $j$ th undermined coefficient. Each of $\Phi_{j}(x)$ of complementary solution sets satisfies the governing equation in Equation (14) as:

$$
\begin{aligned}
& \mathcal{L} \Phi_{1}(x)=0, \mathcal{L} \Phi_{2}(x)=0 \cdots, \\
& \mathcal{L} \Phi_{M-1}(x)=0, \mathcal{L} \Phi_{M}(x)=0
\end{aligned}
$$

Because of the linear property of the differential equation operator in G.E., the potential, $u^{q}(x)$, satisfies the G.E. as:

$$
\begin{aligned}
\mathcal{L} u^{q}(x) & =c_{1} \mathcal{L} \Phi_{1}(x)+c_{2} \mathcal{L} \Phi_{2}(x)+ \\
\cdots & +c_{M-1} \mathcal{L} \Phi_{M-1}(x)+c_{M} \mathcal{L} \Phi_{M}(x)=0
\end{aligned}
$$

It is noted that the potential, $u^{q}(x)$, in the new defined problem is indeed an exact solution because it satisfies the G.E. as shown in Equation (19). In the procedure of numerical algorithm of $M$ number of the undetermined coefficient, $c_{j}$ in the Equation (17), of the quasi-analytic solution of the auxiliary problem, the value of B.C. in the Equations (15) and (16) is specified with the same value of B.C. in the Equations (2) and (3) in the original problem at certain preselected $M_{c}$ number $\left(M_{c} \geq M\right)$ of boundary points. By means of collocation scheme in the auxiliary problem, requiring that the value of quasi-analytic solution at preselected boundary points matches the selected boundary value obtain the numerical value of the undetermined coefficient. It is easy to see that the more boundary points are needed to obtain $M$ number of the undetermined coefficient, $c_{j}$, for an accurate approximation of the real analytical solution. However, a certain $M_{c}$ is implemented due the finite computer precision.

\subsubsection{Modifying the diagonal term}

Because of the same influence matrix between in the original problem and the defined auxiliary problem and the known analytical solution in the computational domain of the auxiliary problem we can modify it by solving the defined auxiliary problem. The process of treatment for modifying diagonal coefficients is presented as follows:

Step 1: By employing the expansion method of RBF in Equation (4), the representation of the solutions in the computational domain, $\Omega$ ', of the defined auxiliary problem is

$$
u^{q}(x)=\sum_{j=1}^{N} A\left(x, s_{j}\right) \alpha_{j}^{q}, x \text { in } \Omega^{\prime}
$$

Step 2: We distribute $N^{\prime}$ number of collocation points, $\left\{x_{i}\right\}_{i=1}^{N^{\prime}}$ in the computational domain, $\Omega^{\prime}$, of the auxiliary problem. The analytical solution, $u^{q}(x)$, of computational domain in the defined auxiliary problem is known in the Equation (14), therefore the known solution at $N^{\prime}$ number of $\left\{x_{i}\right\}_{i=1}^{N^{\prime}}$ is $\left\{u_{i}^{q}\right\}_{i=1}^{N^{\prime}}$. Therefore, the representation of the solutions in the 
Equation (20) at $N^{\prime}$ number of collocation points can be represented as

$$
u_{i}^{q}=\sum_{j=1}^{N} A\left(x_{i}, s_{j}\right) \alpha_{j}^{q}=\sum_{j=1}^{N} A_{i j} \alpha_{j}^{q}, i=1, \ldots, N^{\prime}
$$

The above equations is belong to a linear algebraic equation system with $N^{\prime} \times N$. We can derive the unknown coefficient $\alpha_{j}^{q}$ of the linear algebraic system in Equation (21) by a linear algebraic solver.

Step 3: We can obtain the following equation when the observation point is collocated at the $N$ number of collocation points on the boundary; the location of the $N$ number of collocation points is chosen the same as the original problem, as follows:

$$
u_{i}^{q}=\sum_{j=1, j \neq i}^{N} A_{i j} \alpha_{j}^{q}+\left(A_{i i}+\delta_{i}\right) \alpha_{i}^{q}, i=1, \ldots, N
$$

where $A_{i i}$ is the known diagonal term in the Equation (11), $\delta_{i}$ is the modified coefficient of the $i$ th diagonal terms in the influence matrix, in which it is unknown. The above $N$ number of linear equations is an uncouple system with $N$ number of unknown, $\left\{\delta_{i}\right\}_{i=1}^{N}$, in the Equation (22).

Step 4: We can directly solve each the modified coefficient, $\delta_{i}$, in each equation of the Equation (22) without any difficulty, as follows

$$
\delta_{i}=\frac{\bar{u}_{i}^{q}\left(x_{i}\right)-\sum_{j=1, j \neq i}^{N} A_{i j} \alpha_{j}^{q}}{\alpha_{i}^{q}}-A_{i i}, i=1, \ldots, N
$$

Therefore, we can derive the new $i$ th diagonal term, $A_{i i}+\delta_{i}$, in the Equation (23) to substitute for the origional $i$ th diagonal term of the influence matrix in the Equation (11).

\section{Numerical example}

The case subjected with the Dirchlet B.C. as shown in Fig.1, by having an analytical solution as

$$
u(x, y, z)=e^{y} \sin (z)+x
$$

We solve this case by using the RMM and modified RMM. To see the discrepancy of the value of the diagonal terms for exact value, the diagonal values of the modified RMM and the RMM are shown in Fig.2. The curve of R.M.S error versus the number of source points $N$ is shown in Fig.4. By observing the error curves in Fig.3, the results of the modified RMM are obviously better than the RMM. Finally, we obtain the field solution by using the modified RMM the RMM for $N=2166$ and compare the results with analytical solution as shown in Fig.3 (a)-(c).

\section{Conclusion}

Although the RMM can avoid the major issue in the MFS to choose the best location of a fictitious boundary and it makes the source nodes can be placed on the physical boundary instead at the fictitious boundary, the deficient precision of the solution of RMM is the major issue because of low precision of diagonal value. In this study, we propose a novel technique to modify the diagonal values of the RMM; the numerical results for a numerical example obtained by present scheme are highly acceptable. The proposed technique can thus successfully obtain a more precision solution than the RMM to model 3-D potential problem.

\section{Acknowledgement}

Financial support from the Ministry of Science and Technology of Taiwan, under Grant No: MOST 105-2221-E-197-006 to the National Ilan University is gratefully acknowledged.

\section{References}

D. L. Young, S. J. Jane, C. M. Fan et al., "The method of fundamental solutions for 2D and 3D Stokes problems," Journal of Computational Physics, vol. 211, no. 1, pp. 1-8,(2006).

[2] W. Chen, and F. Wang, "A method of fundamental solutions without fictitious boundary," Engineering Analysis with Boundary Elements, vol. 34, no. 5, pp. 530-532, (2010).

[3] J. Li, Y. C. Hon, and C. S. Chen, "Numerical comparisons of two meshless methods using radial basis functions," Engineering Analysis with Boundary Elements, vol. 26, no. 3, pp. 205225,(2002).

[4] K. H. Chen, C. T. Chen, and J. F. Lee, "Adaptive error estimation technique of the Trefftz method for solving the overspecified boundary value problem," Engineering Analysis with Boundary Elements, vol. 33, no. 7, pp. 966-982, (2009).

[5] D. Young, K. Chen, and C. Lee, "Novel meshless method for solving the potential problems with arbitrary domain," Journal of Computational Physics, vol. 209, no. 1, pp. 290-321, (2005).

[6] W. Chen, J.-Y. Zhang, and Z.-J. Fu, "Singular boundary method for modified Helmholtz equations," Engineering Analysis with Boundary Elements, vol. 44, no. 0, pp. 112-119, (2014).

[7] F. Z. Wang, L. Ling, and W. Chen, 
"Effective condition number for boundary knot method," Computers, Materials \& Continua (CMC), vol. 12, no. 1, pp. 57,(2009).

[8] W. Chen, "Singular boundary method: a novel, simple, meshfree, boundary collocation numerical method," Chinese Journal of Solid Mechanics, vol. 30, no. 6, pp. 592-599, (2009).

[9] K. H. Chen, and J. T. Chen, "Estimating the optimum number of boundary elements by error estimation in a defined auxiliary problem," Engineering Analysis with Boundary Elements, vol. 39, no. 0, pp. 15-22, (2014).

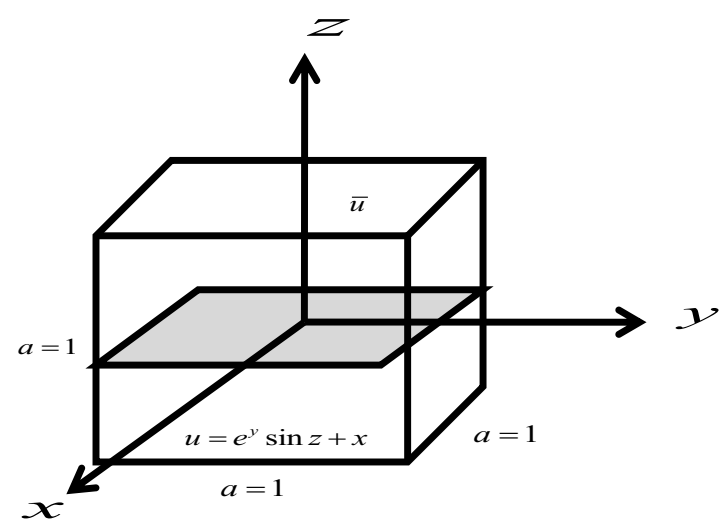

Fig.1 Problem sketch

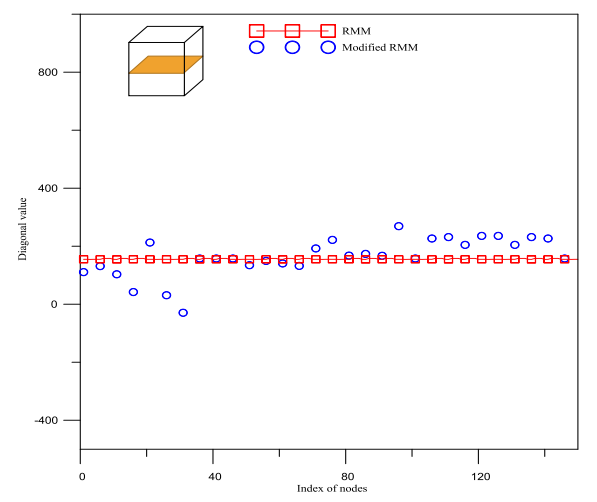

Fig.2 The diagonal coefficients of influence matrix versus the index of nodes

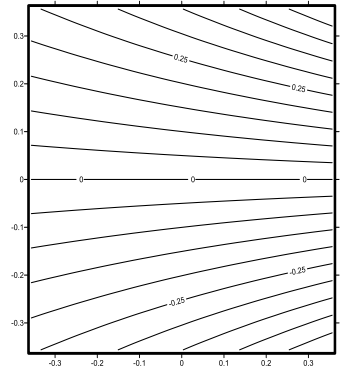

Fig.3 (a) Analytical solution

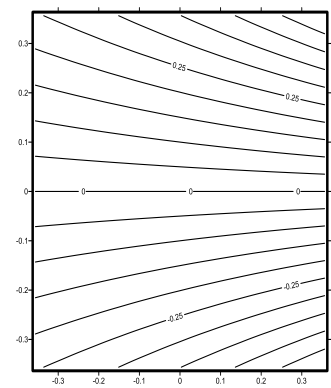

Fig.3 (b) RMM

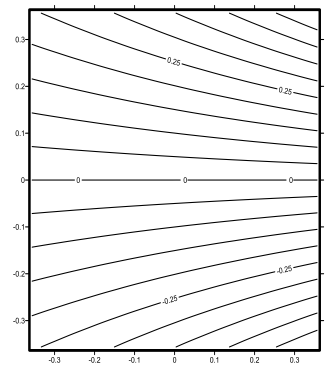

Fig.3 (c) Modified RMM

Fig. 3 The field solution in the $y-z$ plane $(x=0)$, (a) Analytical solution, (b) RMM, (c) Modified

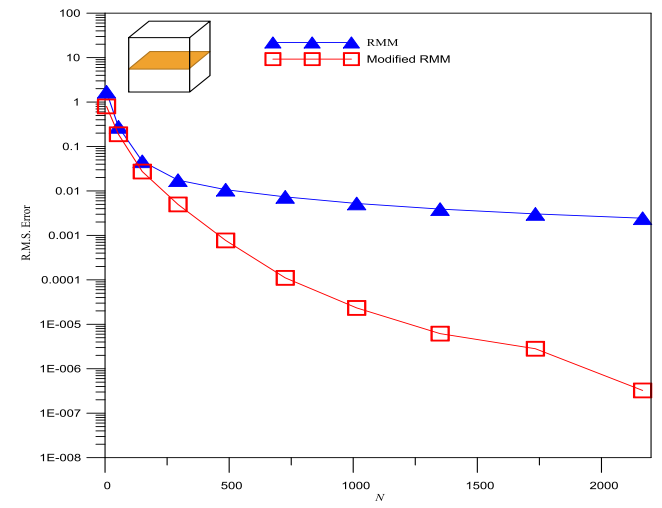

Fig.4 The error analysis versus the number of collocation points 